\title{
Novel method for culturing Schwann cells from adult mouse sciatic nerve in vitro
}

\author{
HAI-BIN WANG $^{1 *}$, XIAO-PAN WANG ${ }^{2 *}$, SHI-ZHEN ZHONG ${ }^{1}$ and ZUN-LI SHEN ${ }^{2}$ \\ ${ }^{1}$ Department of Anatomy, Nanfang Medical University, Guangzhou 510515; ${ }^{2}$ Department Of Plastic Surgery, \\ First Affiliated People's Hospital of Shanghai Jiaotong University, Shanghai 200080, P.R. China
}

Received April 11, 2012; Accepted August 21, 2012

DOI: $10.3892 / \mathrm{mmr} .2012 .1177$

\begin{abstract}
Schwann cells (SCs) are important in the recovery of peripheral nerve injury and are valuable cells for the tissue engineering of artificial neurons. Clinical applications that require pure SCs in large quantities are limited since human and mouse SCs do not attach well to the wall of the culture dish and have low proliferative potential. To obtain high quantities of highly pure SCs, we developed a new method for culturing SCs from the mouse sciatic nerve in vitro. Approximately $1.5 \mathrm{~cm}$ of the bilateral sciatic nerve of a c57 adult mouse was surgically removed and pre-cultured in DMEM containing either 10\% FBS or growth factors. One week later, the in vitro SC culture was observed using light microscopy following enzyme digestion. Cell numbers and cell attachment were examined. The purity of the SCs was determined using s100 $\beta$ and p75NTR staining. Sciatic nerves that had not been pre-cultured were used as the control group. When the excised tissue was precultured in vitro, high yields of SCs were obtained. The SCs were more likely to adhere and the purity was approximately $98 \%$ at the 1 generation following simple purification steps, which was significantly higher than the purity obtained from the control group. The pre-culturing of the sciatic nerve prior to in vitro tissue culturing significantly increased the quantity and quality of the SCs.
\end{abstract}

\section{Introduction}

Schwann cells (SCs) are the principal cells of the peripheral nervous system. They often serve as the seed cells for the tissue

Correspondence to: Professor Zun-Li Shen, Department Of Plastic Surgery, First Affiliated People's Hospital of Shanghai Jiaotong University, 100 Haining Road, Hongkou District, Shanghai 200080, P.R. China

E-mail: zhunli_shen@yahoo.com.cn

${ }^{*}$ Contributed equally

Key words: adult mice, sciatic nerve, Schwann cells, in vitro tissue culture, growth factor engineering of artificial neurons and contribute significantly to the recovery from peripheral nerve injury $(1,2)$. SCs ensure favorable conditions for the regeneration of axons and guide the distal growth of axons by secreting the corresponding nerve growth factors and extracellular matrix (3). However, as end-stage cells, directly derived SC lines do not attach well and have low proliferative potential; therefore, clinical applications that require pure SCs in large quantities are limited. It has been shown that SCs express various markers during different stages of development and injury repair processes, indicating that SCs have proliferative potential that varies according to context and are not functionally and structurally homogeneous. In this regard, to further expand the scope of the clinical applications of SCs, we introduce in this study a new approach for the culturing of SCs from the adult mouse in vitro.

\section{Materials and methods}

Animals. A total of 15 clean 6- to 8-week-old c57 mice were provided by the Shanghai Silaike Experimental Animal Center. The study was approved by the ethics committee of Shanghai Jiaotong University

Reagents and equipment. DMEM and FBS were purchased from Hyclone (Shanghai, China). Forskolin was obtained from Sigma (St. Louis, MO, USA). Heregulin- $\beta-1$ and basic-FGF (b-FGF) were from Peprotech, Inc. (Rocky Hill, NJ, USA). Collagenase NB4 (neutral protease, grade II) was from Roche Diagnostics GmbH (Mannheim, Germany). Dispase was purchased from Serva (Heidelberg, Germany). The multiclonal s100 $\beta$ rabbit antibody and fluorescent mounting medium were from Dako (Ely, UK). The multiclonal p75NTR rabbit antibody was from Abcam (Cambridge, UK). The multiclonal goat antirabbit IgG-rhodamine was from Santa Cruz Biotechnology, Inc. (Santa Cruz, CA, USA).

Medium containing growth factors. Schwann cell culture medium (SCCM) was prepared by supplementing DMEM with $10 \%$ FBS, $2 \mu \mathrm{M}$ forskolin, $10 \mathrm{ng} / \mathrm{ml}$ heregulin- $\beta-1$ and $50 \mathrm{ng} / \mathrm{ml}$ b-FGF.

Poly-lysine coating. Culture dishes were covered with $0.1 \mathrm{mg} / \mathrm{ml}$ poly-lysine working solution, incubated at $37^{\circ} \mathrm{C}$ for 
$1 \mathrm{~h}$, washed with double-distilled water and refrigerated at $4^{\circ} \mathrm{C}$ until use.

Animal surgery and in vitro tissue culture. Adult c57 mice were sacrificed by cervical dislocation and surface sterilized in $75 \%$ ethanol for $10 \mathrm{~min}$. Both sides of the sciatic nerves were surgically exposed on a clean bench and a $1.5-\mathrm{cm}$ fragment was removed for use. The skin was stripped under a dissection scope (Motic, Xiameng, China) and the sciatic nerves were rinsed with PBS and placed in DMEM supplemented with $10 \%$ FBS or SCCM in a 6-well plate. The plate was incubated at $37^{\circ} \mathrm{C}$ under $5 \% \mathrm{CO}_{2}$ in a $\mathrm{CO}_{2}$ incubator (Forma Scientific, Inc., Marietta, $\mathrm{OH}, \mathrm{USA}$ ) for one week. The media were changed once every two days.

Cell culture and purification. Fresh sciatic nerves and the sciatic nerves that had been incubated in DMEM with $10 \%$ FBS or SCCM were rinsed with PBS, cut into 2-mm pieces and digested with a mixture of $0.2 \%$ collagenase NB4 and $0.2 \%$ dispase at $37^{\circ} \mathrm{C}$. The digested materials were then centrifuged at $1,500 \mathrm{rpm}$ for $5 \mathrm{~min}$ and the supernatants were discarded. The pellets were resuspended in SCCM and replated in poly-lysine coated dishes following cell counting. The dishes were incubated at $37^{\circ} \mathrm{C}$ under $5 \% \mathrm{CO}_{2}$. The cells obtained from the SCCM cultured nerves were purified following $48 \mathrm{~h}$ of incubation (4).

Immunostaining with s100 $\beta$ and p75NTR. S100 $\beta$ and p75NTR immunostaining was performed for $\mathrm{p} 0$ and $\mathrm{p} 1$ cells as follows. The cells were fixed with $4 \%$ paraformaldehyde and membranes were permeabilized with $0.3 \%$ Triton X-100. The samples were then blocked with goat serum at $37^{\circ} \mathrm{C}$ for $30 \mathrm{~min}$, incubated with primary antibody at $37^{\circ} \mathrm{C}$ for $1 \mathrm{~h}$, washed for 5 min with PBS three times and incubated with secondary antibody at $37^{\circ} \mathrm{C}$ for $30 \mathrm{~min}$. Finally, the cell nuclei were stained with DAPI. Images were captured under a fluorescent microscope (Nikon, Tokyo, Japan). Cells in three randomly selected fields were counted for SC purity calculations.

Frozen section and s $100 \beta$ immunostaining. Fresh sciatic nerves and the sciatic nerves that had been incubated in DMEM with $10 \%$ FBS or SCCM were fixed, dehydrated and frozen-sliced into $8-\mu \mathrm{m}$ sections. Immunostaining and DAPI staining were performed as described in the previous section. Samples were observed and images captured under a confocal microscope.

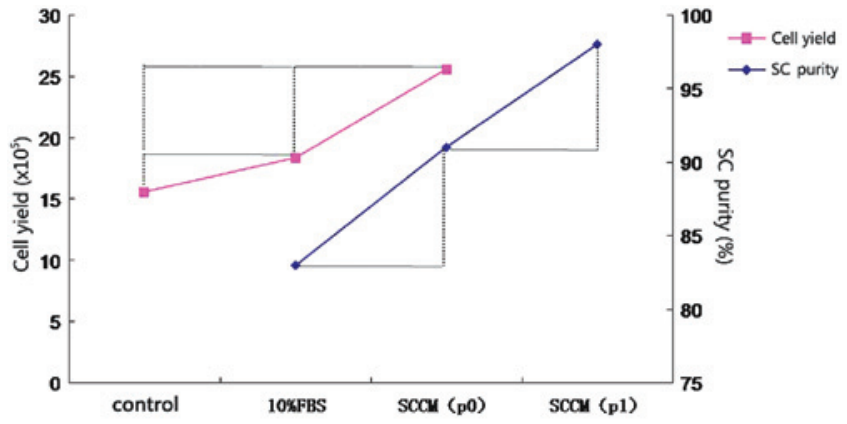

Figure 1. Cell number before inoculation in various groups and the cell purities of primary, SCCM p0 and SCCM p1 (after purification) generations. SCs, Schwann cells; SCCM, SC-conditioned medium.

Statistical analysis. All data are expressed as the average \pm standard deviation $(\mathrm{x} \pm \mathrm{s})$. Statistical analyses were performed using the SPSS 13.0 software. Comparisons between the averages of the groups were evaluated using the Student's t-test. $\mathrm{P}<0.05$ was considered to indicate a statistically significant result.

\section{Results}

Cell counting. Cell suspensions $(20 \mu \mathrm{l})$ for the various groups were counted using a hemacytometer prior to inoculation. The cell count for the SCCM group $\left(2.56 \times 10^{6} / 3 \mathrm{~N}\right)$ was significantly higher than the counts for the control $\left(1.56 \times 10^{6} / 3 \mathrm{~N}\right)$ and the DMEM with $10 \%$ FBS groups $\left(1.84 \times 10^{6} / 3 \mathrm{~N}\right)$ (Fig. 1).

Observation of cell growth. At $48 \mathrm{~h}$ after inoculation, the attachment of the cells was observed under an inverse microscope. Two types of cells with different morphologies were obtained. SCs were small and appeared in long fusiform, bipolar or tripolar shapes with strong refraction while fibroblasts were large and appeared in flat multilateral or irregular shapes with weak refraction. The uncultured cells which remained suspended were dead. Only a small fraction of the cells attached and proliferated. The majority of the cells derived from the sciatic nerves cultured in DMEM with $10 \%$ FBS were observed to be attached, however a high percentage of the cells were fibroblasts. The cells derived from the sciatic nerves cultured in SCCM attached and only a few of them were fibroblasts (Fig. 2).
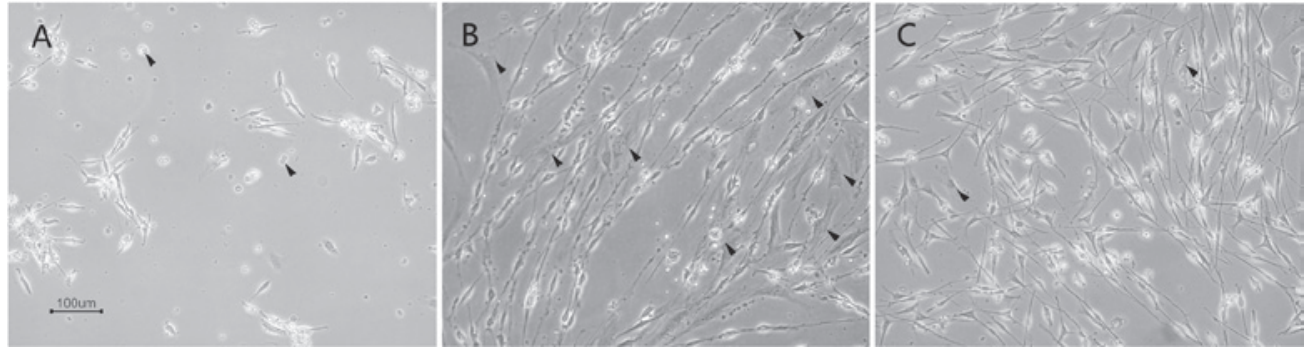

Figure 2. Primary cells in various groups following $48 \mathrm{~h}$ of incubation (magnification, x100). (A) Cells derived from uncultured sciatic nerves. The arrow indicates a suspended dead cell. (B) Cells derived from sciatic nerves cultured in DMEM with 10\% FBS. The majority of the cells attached, but a high percentage of the cells were fibroblasts (indicated by arrows). (C) Cells derived from sciatic nerves cultured in SCCM. Cells were attached and only a few cells were fibroblasts. SCCM, Schwann cell-conditioned medium. 

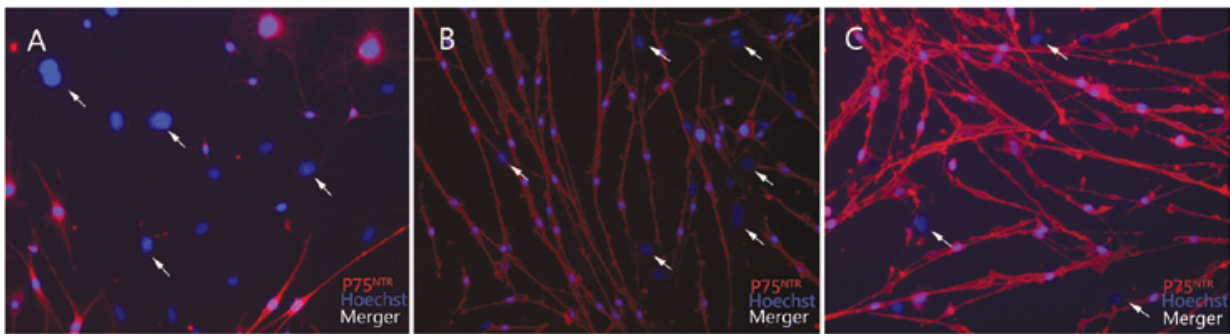

Figure 3. p75NTR immunostaining of primary cells in various groups $48 \mathrm{~h}$ after cultivation (magnification, x100). The bipolar and tripolar SCs in (A and B) were all stained positive for p75NTR. (A) Cells derived from uncultured sciatic nerves. Few cells were SCs and the majority of cells were fibroblasts that stained negative. (B) Cells derived from sciatic nerves cultured in DMEM with 10\% FBS. Both SCs and fibroblasts were abundant. (C) Cells derived from sciatic nerves cultured in SCCM. Few fibroblasts were present. Arrows indicate fibroblasts that were stained negative for p75NTR. SCs, Schwann cells; SCCM, SC-conditioned medium.

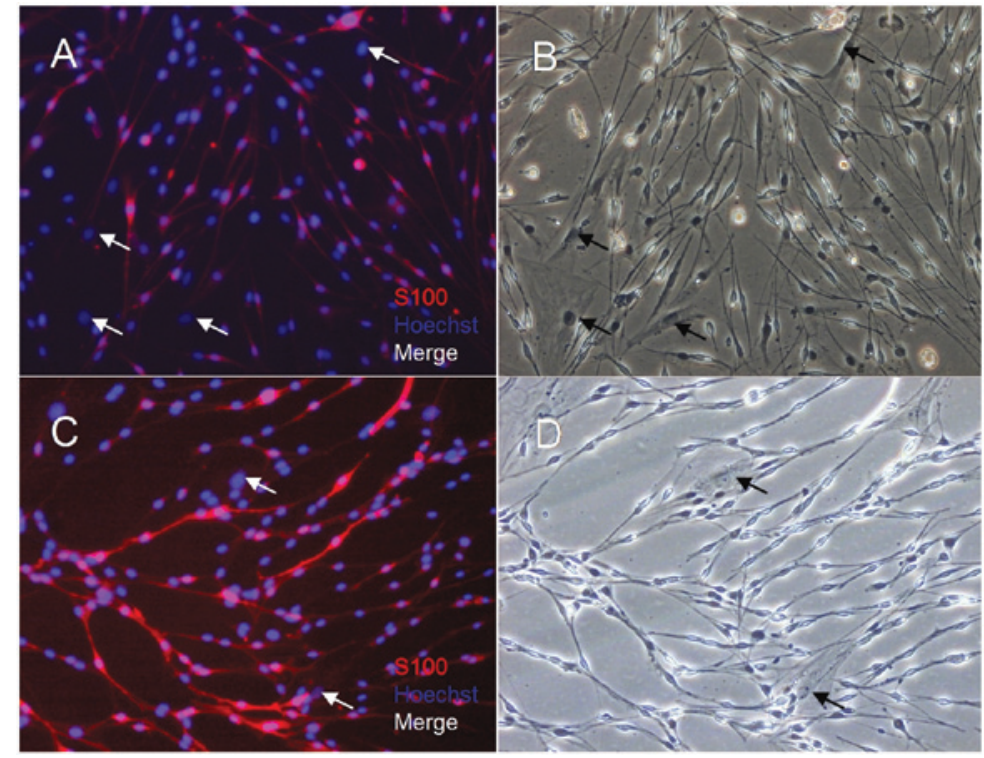

Figure 4. s100 $\beta$ immunostaining of cells derived from sciatic nerves cultured in SCCM (p0) and cells after purification (p1) (magnification, x100). The bipolar and tripolar SCs in p0 (A) and p1 (C) were all stained positive, while irregular and flat fibroblasts were stained negative for s100 $\beta$. Few fibroblasts were present in p1 and the purity of the SCs was high. (B and D) are corresponding bright field pictures of (A and C), respectively. SCs, Schwann cells; SCCM, SC-conditioned medium.

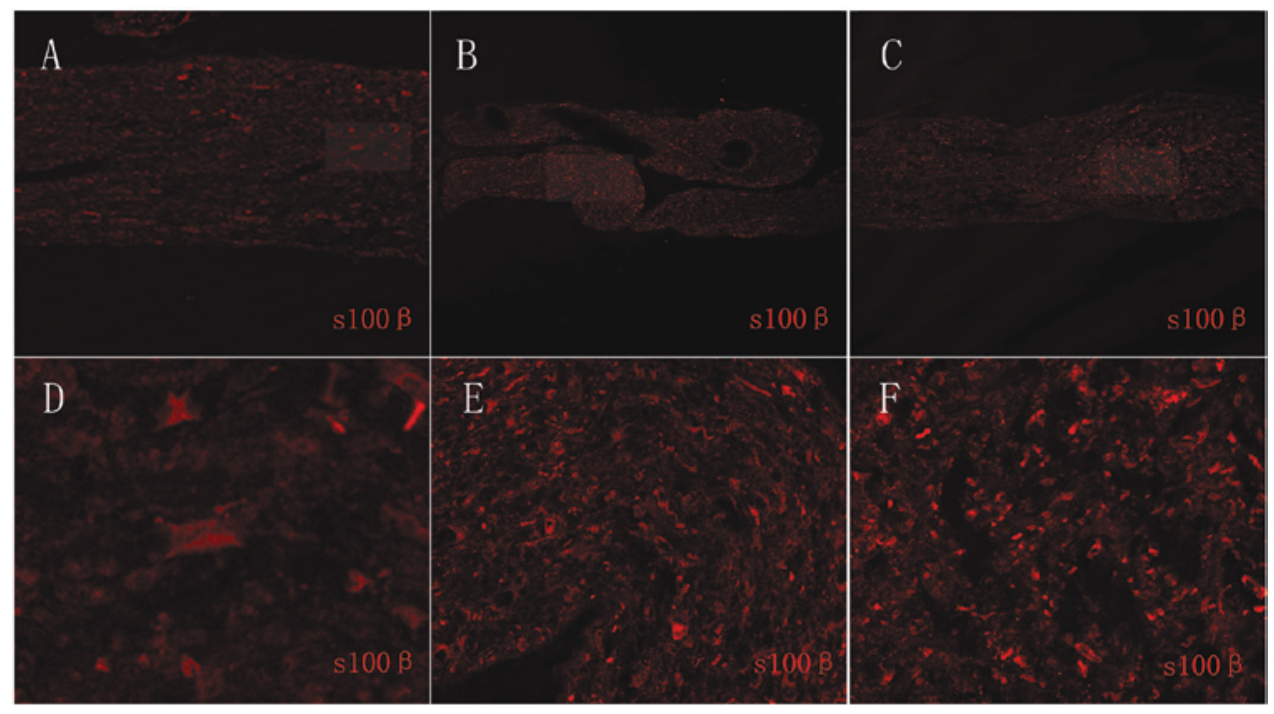

Figure 5. Histological section of s100 $\beta$ immunostaining samples of various groups observed under a confocal microsope. (A-C) are cells derived from fresh sciatic nerves, sciatic nerves cultured in DMEM with $10 \%$ FBS and sciatic nerves cultured in SCCM, respectively. (D-F) are enlargements of the shadowed areas in (A-C), respectively. SCs derived from sciatic nerves cultured in SCCM proliferated and formed bands of Büngner. SCs, Schwann cells; SCCM, SC-conditioned medium. 
Immunostaining and cell purity determination. After $48 \mathrm{~h}$ of incubation, cells from the various groups were stained with anti-p75NTR antibody. Almost all bipolar or tripolar cells stained positive, while the fibroblasts stained negative (Fig. 3). The purities of the SCs from the DMEM with 10\% FBS group and the SCCM group were 83 and 91\%, respectively (Fig. 1). The purity of the SCs was not determined for cells derived from fresh sciatic nerves since the cells were not attached, even following $72 \mathrm{~h}$ of incubation.

Following one simple purification (p1) step for the cells derived from the sciatic nerves cultured in SCCM, almost all bipolar and tripolar cells were stained positive for $s 100 \beta$ (Fig. 4). The purity of the SCs was 98\% (Fig. 1).

Histological section of s100 $\beta$ immunostaining samples. Cells derived from the sciatic nerves cultured in SCCM attached well and resulted in high yields of highly pure SCs. Therefore, we investigated the changes in the SCs at the tissue level. The SCs from the sciatic nerves cultured in SCCM proliferated and formed bands of Büngner, which is similar to the Wallerian degeneration process following nerve injury. The SCs from sciatic nerves cultured in DMEM with $10 \%$ FBS proliferated but did not form bands of Büngner. The SCs from fresh sciatic nerves did not proliferate (Fig. 5).

\section{Discussion}

The development of neural tissue engineering provides a new route for peripheral nerve repair. SCs are seed cells for neural tissue engineering and contribute significantly to the repair of injured peripheral nerves. However, as end-stage cells, SCs are difficult to culture and are usually obtained in poor yields and low purities. In addition, despite the efforts of many scientists to induce Schwann-like cells from stem cells (5-7), further applications of these methods are limited due to low induction rates, dedifferentiation following the removal of the inducing agents and the tumor-prone properties of the induced cells (8).

To obtain high quantities of high quality SCs, the in vitro culturing and proliferation of SCs has been extensively used. Previous studies have shown that SCs have different proliferative potentials at different developmental stages and are not functionally and structurally homogeneous. During the Wallerian degeneration of nerve injury, SCs dedifferentiate, express neurotrophic factors, cell adhesion molecules $(9,10)$ and the immature SC marker glial fibrillary acidic protein (GFAP) and upregulate p75NTR (11-13). These may facilitate SC proliferation and attachment and promote nerve regeneration. In 1999, Keilhoff et al (14) obtained large quantities of SCs with high proliferative potential following in vivo pre-degeneration (Wallerian degeneration) for a week and in vitro recultivation. However, in vivo pre-degeneration requires two surgical procedures, is time-consuming and causes a delay in treatment. The clinical applications of this approach are also limited due to individual differences and the resulting difficulties in estimating the timing of the desired pre-degeneration effect. In our study, the sciatic nerves of adult mice were pre-degenerated in vitro in media that mimicked in vivo conditions. In 2010, Kraus et al (15) reported an in vitro pre-degeneration process in which sciatic nerves were incubated in DMEM with $10 \%$ FBS for one week and large quantities of pure SCs were obtained. Based on these advances, we included in our medium forskolin, heregulin- $\beta-1$ and b-FGF (SCCM) to promote the growth of the SCs. The experimental conditions used by Kraus et al were used for our positive control. After one week, the SC count in the SCCM group was $2.56 \times 10^{6} / 3 \mathrm{~N}$, which was significantly higher than the counts in the DMEM with $10 \%$ FBS group and the uncultured group (Fig. 1). When viewed 48 h post-inoculation, many cells attached and these cells were confirmed to be SCs by immunostaining. Only small amounts of fibroblasts were present. The purity of the SCs in p0 was 91\%, compared with $83 \%$ purity in the DMEM with $10 \%$ FBS group. Following one simple purification step, the purity reached $98 \%$. In 1995, Dong et al (16) described the concentration-dependent death of in vitro cultured cells - the cells died more readily at low concentrations. Cells cultured in vitro secrete mitosispromoting growth factors (17) and cell-cell contacts not only further enhance the release of these factors to induce cell division and proliferation (18) but also suppress the growth of fibroblasts.

We also investigated the proliferation of SCs induced by forskolin, heregulin- $\beta-1$ and $b-F G F$ at the tissue level. Forskolin, heregulin- $\beta-1$ and b-FGF stimulated the proliferation of SCs, which formed bands of Büngner and promoted the dedifferentiation of SCs. These effects were not significant in the uncultured and the DMEM with $10 \%$ FBS groups.

We have developed, in this study, a method for cultivating a large number of pure SCs in vitro. This finding helps to fulfill the need to develop artificial neurons and further enriches SC research and the possible clinical applications of SCs.

\section{References}

1. Meek MF and Coert JH: Clinical use of nerve conduits in peripheral nerve repair:reiew of literature. J Reconstr Microsurg 18: 97-109, 2002.

2. Shen ZL, Berger A, Hierner R, et al: A Schwann cell-seeded intrinsic framework and its satisfactory biocompatibility for a bioartificial nerve graft. Microsurgery 21: 6-11, 2001.

3. Frostick SP, Yin Q and Kemp GJ: Schwann cells, neurotrophic factors, and peripheral nerve regeneration. Microsurgery 18: 397-405, 1998.

4. Jin YQ, Liu W, Hong TH and Cao Y: Efficient Schwann cell purification by differential cell detachment using multiplex collagenase treatment. J Neurosci Methods 170: 140-148, 2008.

5. Dezawa M, Takahashi I, Esaki M, et al: Sciatic nerve regeneration in rats induced by transplantation of in vitro differentiated bone-marrow stromal cells. Eur J Neurosci 14: 1771-1776, 2001.

6. Kingham PJ, Kalbermatten DF, Mahay D, et al: Adipose-derived stem cells differentiate into a Schwann cell phenotype and promote neurite outgrowth in vitro. Exp Neurol 207: 267-274, 2007.

7. Jiang L, Zhu JK, Liu XL, et al: Phenotypic and functional characteristics of rat adipose tissue-derived stromal cells differentiated into Schwann-like cells in vitro. Chin J Microsurgery 30: 430-432, 2007 (In Chinese).

8. Cogle CR, Theise ND, Fu D, et al: Bone marrow contributes to epithelial cancers in mice and humans as developmental mimicry. Stem Cells 25: 1881-1887, 2007.

9. Bolin LM, Iismaa TP and Shooter EM: Isolation of activated adult Schwann cells and a spontaneously immortal Schwann cell clone. J Neurosci Res 33: 231-238, 1992.

10. Fannon AM, Sherman DL, Ilyina-Gragerova G, et al: Novel E-cadherin mediated adhesion in peripheral nerve: Schwann cell architecture is stabilized by autotypic adherens junctions. J Cell Biol 129: 189-202, 1995. 
11. Jessen KR and Mirsky R: Negative regulation of myelination: Relevance for development, injury, and demyelinating disease. Glia 56: 1552-1565, 2008 .

12. Lee HK, Seo IA, Suh DJ, et al: Interleukin-6 is required for the early induction of glial fibrillary acidic protein in Schwann cells during Wallerian degeneration. J Neurochem 108: 776-786, 2009.

13. Lemke $\mathrm{G}$ and Chao M: Axons regulate Schwann cell expression of the major myelin and NGF receptor genes. Development 102: 499-504, 1988.

14. Keilhoff G, Fansa H, Schneider W, et al: In vivo predegeneration of peripheral nerves: an effective technique to obtain activated Schwann cells for nerve conduits. J Neurosci Methods 89: 17-24, 1999.
15. Kraus A, Täger J, Kohler K, et al: Efficacy of various durations of in vitro predegeneration on the cell count and purity of rat Schwann-cell cultures. J Neurotrauma 27: 197-203, 2010.

16. Dong Z, Brennan A, Liu N, et al: Neu differentiation factor is a neuron-glia signal and regulates survival, proliferation, and maturation of rat Schwann cell precursors. Neuron 15: 585-596, 1995.

17. Eccleston PA, Collarini EJ, Jessen KR, et al: Schwann cells secrete a PDGF-like factor: evidence for an autocrine growth mechanism involving PDGF. Eur J Neurosci 2: 985-992, 1990.

18. Sasagasako N, Toda K, Hollis M and Quarles RH: Myelin gene expression in immortalized Schwann cells: relationship to cell density and proliferation. J Neurochem 66: 1432-1439, 1996. 